\title{
THE EQUIVALENT ELASTIC COMPLIANCES OF AN ELASTIC SOLID WITH INTERFACE CRACKS
}

\author{
Xiaoping Lu and Maria Comninou \\ Department of Mechanical Engineering and Applied Mechanics, University of Michigan, Ann Arbor, \\ MI 48109-2125, U.S.A.
}

(Received 5 December 1989; and in revised form 28 June 1990)

\begin{abstract}
A periodic array of interface cracks was considered earlier as a simple model to study the effect of crack interaction on the elastic fields of a solid composed of two dissimilar isotropic elastic materials. This paper focuses on the perturbation of the elastic compliances of the solid due to the interacting interface cracks. The problem is formulated using Betti's reciprocal theorem. The analysis provides the equivalent elastic compliances in terms of the geometric parameters of the interface cracks for various levels of loadings and material combinations. The results show that the interaction between cracks is significant only when the crack spacing is less than four times the crack length. Also, contact between crack faces introduces coupling between applied shear stress and normal displacement, but it is significant only when the ratio of the magnitudes of the shear and normal stresses exceeds two.
\end{abstract}

\section{INTRODUCTION}

The widespread use of composite materials in structural applications has generated renewed interest in problems involving interfaces between dissimilar media, including the classical problem of the interface crack $[1,2]$. Modern composites, particularly those involving ceramics [3], frequently consist of brittle fibers in a brittle matrix and they therefore rely for their ductility on frictional dissipation at the fiber/matrix interface. When such composites are loaded monotonically, their behavior remains linear until cracks begin to develop, usually either in the matrix material or at the fiber/matrix interface. These cracks reduce the incremental stiffness of the composite, resulting in a concave downward stress-strain curve.

Conversely, when a brittle composite exhibits such a stress-strain curve, it is an indication of the generation of cracks within the structure, and in principle it should be possible to estimate the crack density from the incremental stiffness of the bulk material.

The additional compliance of a composite specimen due to a system of cracks will depend on the typical crack size and spacing and on whether the cracks occur in the matrix or at the fiber/matrix interface. In the case of interface cracks, a further complication is introduced through the tendency of the cracks to close when the far-field stresses involve a significant shear component [4]. This closure introduces a measure of coupling between the effects of normal and shear loading and also leads to a nonlinear effective constitutive law, since the normal displacements associated with shear will have the same sign for both directions of shear loading.

In the present paper, we investigate these effects by determining the additional compliance of an infinite body of two bonded dissimilar materials due to a periodic array of cracks at the interface. The results are obtained by extending the solution due to Schmueser and Comninou [6], in which the perturbed elastic field is expressed in terms of a distribution of edge dislocations along the cracks. The additional compliance is obtained from these dislocation distributions using Betti's reciprocal theorem. Numerical results are given for various ratios of tension to shear stress in the far-field.

\section{FORMULATION}

The geometry and formulation are the same as in [6]. However, in the interests of convenience and continuity, we repeat the essential steps of the analysis. 


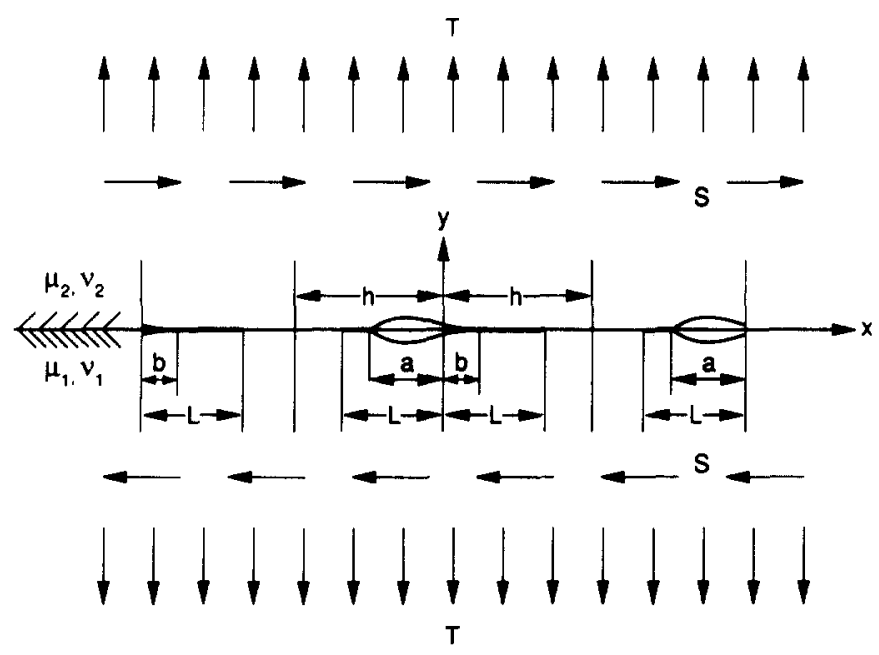

FIG. 1. The periodic array of interface cracks.

Consider a periodic array of interface cracks with period $2 h$, lying at the interface between two elastic solids characterized by shear moduli $\mu_{1}, \mu_{2}$, and Poisson's ratios $v_{1}, v_{2}$, as shown in Fig. 1. Under the action of uniform shear stress $S$ and tension $T$ applied at infinity, a representative crack of length $2 L$ opens over an interval $(-a, b)$; its two faces are in frictionless contact at the crack tips over the intervals $(-L,-a)$ and $(b, L)$. The parameters $a$ and $b$ are to be determined from the traction boundary conditions, which require that shear tractions vanish over the entire length of each crack and that normal tractions vanish over the open part of each crack. The conditions are enforced by using the known stress fields for edge dislocations.

Periodic dislocation distributions $B_{x}(x)$ and $B_{y}(x)$ are defined over the entire length and over the open part of each crack, respectively. The boundary conditions at the interface are given in terms of the applied field and a corrective field determined by the dislocation distributions:

$$
S+C\left[\beta B_{y}(x)-\frac{1}{\pi} \int_{-\infty}^{\infty} \frac{B_{x}(\xi)}{\xi-x} \mathrm{~d} \xi\right]=0,
$$

over the entire length of each crack, and

$$
T-C\left[\beta B_{x}(x)+\frac{1}{\pi} \int_{-\infty}^{\infty} \frac{B_{y}(\xi)}{\xi-x} \mathrm{~d} \xi\right]=0,
$$

over the open part of each crack, where

$$
\begin{aligned}
& C=\frac{2 \mu_{1}(1+\alpha)}{\left(\kappa_{1}+1\right)\left(1-\beta^{2}\right)}, \\
& \alpha=\frac{\mu_{2}\left(\kappa_{1}+1\right)-\mu_{1}\left(\kappa_{2}+1\right)}{\mu_{2}\left(\kappa_{1}+1\right)+\mu_{1}\left(\kappa_{2}+1\right)}, \\
& \beta=\frac{\mu_{2}\left(\kappa_{1}-1\right)-\mu_{1}\left(\kappa_{2}-1\right)}{\mu_{2}\left(\kappa_{1}+1\right)+\mu_{1}\left(\kappa_{2}+1\right)} .
\end{aligned}
$$

Taking periodicity into account, equations (1) and (2) become:

$$
\begin{aligned}
& S+C\left\{\beta B_{y}(x)-\frac{1}{2 h} \int_{-L}^{L} B_{x}(\xi) \cot \left[\frac{\pi(\xi-x)}{2 h}\right] \mathrm{d} \xi\right\}=0, \quad-L<x<L \\
& T-C\left\{\beta B_{x}(x)-\frac{1}{2 h} \int_{-a}^{b} B_{y}(\xi) \cot \left[\frac{\pi(\xi-x)}{2 h}\right] \mathrm{d} \xi\right\}=0, \quad-a<x<b .
\end{aligned}
$$


To ensure single-valued displacements, the following conditions are applied:

$$
\begin{gathered}
\int_{-L}^{L} B_{x}(\xi) \mathrm{d} \xi=0 \\
\int_{-a}^{b} B_{y}(\xi) \mathrm{d} \xi=0 .
\end{gathered}
$$

The known displacement fields of the dislocation distributions given in [6] can be used to determine the relative displacements between the solids; however, the process is cumbersome. Therefore, Betti's reciprocal theorem [7] is employed to obtain the expressions for the relative displacements. The theorem states that, for a linear elastic body subjected to two different loadings, the work done by the first loading acting through the displacements produced by the second loading, equals the work done by the second loading acting through the displacements produced by the first loading. That is,

$$
\int_{S} T_{i}^{(1)} u_{i}^{(2)} \mathrm{d} S+\int_{V} F_{i}^{(1)} u_{i}^{(2)} \mathrm{d} V=\int_{S} T_{i}^{(2)} u_{i}^{(1)} \mathrm{d} S+\int_{V} F_{i}^{(2)} u_{i}^{(1)} \mathrm{d} V
$$

where $T_{i}^{(k)}$ stands for surface traction, $F_{i}^{(k)}$ for body force, and $u_{i}^{(k)}$ for the displacement produced by the corresponding loading condition; the superscripts identify the quantities of a given loading, and the subscripts identify the quantities in the $x_{i}$ direction.

In the absence of body forces, the above equation becomes:

$$
\int_{S} T_{i}^{(1)} u_{i}^{(2)} \mathrm{d} S=\int_{S} T_{i}^{(2)} u_{i}^{(1)} \mathrm{d} S
$$

To obtain the relative normal displacement for the current problem, consider the stress state of uniform tension $\sigma_{0}$ throughout the solid, and the auxiliary stress state defined by the corrective solution of the current problem. For the state of the uniform stress the displacements at the crack faces are zero, while for the auxiliary state the stress components at infinity are zero. Thus, Betti's reciprocal theorem gives the following equation:

$$
\sigma_{0} \int_{-h}^{h} U_{y}^{1} \mathrm{~d} x-\sigma_{0} \int_{-h}^{h} U_{y}^{2} \mathrm{~d} x-\sigma_{0} \int_{-a}^{b} u_{y}^{2}(x, 0) \mathrm{d} x+\sigma_{0} \int_{-a}^{b} u_{y}^{1}(x, 0) \mathrm{d} x=0
$$

where $U_{y}^{1}$ and $U_{y}^{2}$ are the normal displacements at infinity, $u_{y}^{1}$ and $u_{y}^{2}$ are the normal displacements along the crack faces produced by the dislocation distribution along the cracks. The superscripts 1 and 2 stand for solids 1 and 2, respectively. Because of the periodicity, only the work done on one crack needs to be considered.

Denoting the average relative normal displacement at infinity by $\delta_{n}$

$$
\delta_{n}=\frac{1}{2 h} \int_{-h}^{h}\left(U_{y}^{1}-U_{y}^{2}\right) \mathrm{d} x
$$

we obtain from equation (9):

where

$$
\delta_{n}=\frac{1}{2 h} \int_{-a}^{b} g(x) \mathrm{d} x
$$

$$
g(x)=u_{y}^{2}(x, 0)-u_{y}^{1}(x, 0)
$$

is the crack opening displacement related to the component of the Burgers vector $B_{y}(x)$ by

$$
B_{y}(x)=-\frac{\mathrm{d} g(x)}{\mathrm{d} x}
$$

Therefore, the average normal displacement at infinity is equal to the average crack opening at the crack faces, and

$$
\delta_{n}=-\frac{1}{2 h} \int_{-a}^{b} \int_{-a}^{x} B_{y}(\xi) \mathrm{d} \xi \mathrm{d} x
$$


Similarly, to obtain the relative tangential displacement, consider the state of simple shear stress $\sigma_{0}$ throughout the solid, and the auxiliary state of stress given by the corrective solution. Equation (8) then gives:

$$
\sigma_{0} \int_{-h}^{h} U_{x}^{1} \mathrm{~d} x-\sigma_{0} \int_{-h}^{h} U_{x}^{2} \mathrm{~d} x-\sigma_{0} \int_{-L}^{L} u_{x}^{2}(x, 0) \mathrm{d} x+\sigma_{0} \int_{-L}^{L} u_{x}^{1}(x, 0) \mathrm{d} x=0
$$

where the subscript $x$ indicates the quantities in the tangential direction.

Therefore, the average relative tangential displacement, $\delta_{t}$, is obtained as:

$$
\begin{aligned}
\delta_{t} & =\frac{1}{2 h} \int_{-h}^{h}\left(U_{x}^{1}-U_{x}^{2}\right) \mathrm{d} x \\
& =\frac{1}{2 h} \int_{-L}^{L} h(x) \mathrm{d} x
\end{aligned}
$$

where

$$
h(x)=u_{x}^{2}(x, 0)-u_{x}^{1}(x, 0)
$$

is the relative slip between the two solids at the crack faces related to the dislocation component $B_{x}(x)$ by

$$
B_{x}(x)=-\frac{\mathrm{d} h(x)}{\mathrm{d} x}
$$

Thus, the average shear displacement at infinity is equal to the average relative slip between two solids at the crack faces, and

$$
\delta_{t}=-\frac{1}{2 h} \int_{-L}^{L} \int_{-L}^{x} B_{x}(\xi) \mathrm{d} \xi \mathrm{d} x
$$

To calculate the displacements $\delta_{n}$ and $\delta_{t}$, we need to recalculate the dislocation components $B_{x}(x), B_{y}(x)$, and the contact zones $a$ and $b$ from the integral equations (3)-(6). Equations (3) and (4) can be transformed to equations with explicit Cauchy kernels by means of the following changes of variables as in [6]:

where

$$
\begin{aligned}
x & =L x^{\prime}, \quad \xi=L \xi^{\prime} \\
\tan \left(\frac{\pi L \xi^{\prime}}{2 h}\right) & =u d, \quad \tan \left(\frac{\pi L x^{\prime}}{2 h}\right)=s d
\end{aligned}
$$

$$
\begin{aligned}
d & =\tan \left(\frac{\pi L}{2 h}\right) \\
\gamma_{1} & =-\frac{1}{d} \tan \left(\frac{\pi a}{2 h}\right), \quad \gamma_{2}=\frac{1}{d} \tan \left(\frac{\pi b}{2 h}\right)
\end{aligned}
$$

and the same symbols were retained in the new variables for functions $B_{x}$ and $B_{y}$.

The relative displacements in the new variables are:

where

$$
\begin{aligned}
& \delta_{n}=-\frac{2 d^{2} h}{\pi^{2}} \int_{\gamma_{1}}^{\gamma_{2}} \frac{1}{1+d^{2} s^{2}} \int_{\gamma_{1}}^{s} \phi(u) \mathrm{d} u \\
& \delta_{t}=-\frac{2 d^{2} h}{\pi^{2}} \int_{-1}^{1} \frac{1}{1+d^{2} s^{2}} \int_{-1}^{s} \psi(u) \mathrm{d} u
\end{aligned}
$$

$$
\begin{aligned}
& \psi(u)=\frac{B_{x}(u)}{1+d^{2} u^{2}}, \\
& \phi(u)=\frac{B_{y}(u)}{1+d^{2} u^{2}} .
\end{aligned}
$$

Details of determination of $\psi, \phi, \gamma_{1}$ and $\gamma_{2}$ are given in [6]. 


\section{RESULTS AND DISCUSSION}

Most of the numerical results were generated for $\beta=0.5$ because this case corresponds to one material being rigid and has the biggest effect on the compliances. Some results are also given for $\beta=0,0.3$ for comparison. The results are presented for various values of the dimensionless loading parameter $T / S$ and the crack configuration parameter $h / L$. Both tensile and compressive loading conditions were analysed.

Figures 2 and 3 show the variation of the dimensionless normal displacement $\delta_{n} C / S L$ with ratios of crack length $L$ to spacing $h$, for tension/shear loading and compression/shear loading, respectively. From these figures it is seen that $\delta_{n} C / S L$ is proportional to $L / h$ for $L / h$ less than 0.25 , and it then increases rapidly with increasing $L / h$. This indicates that there is

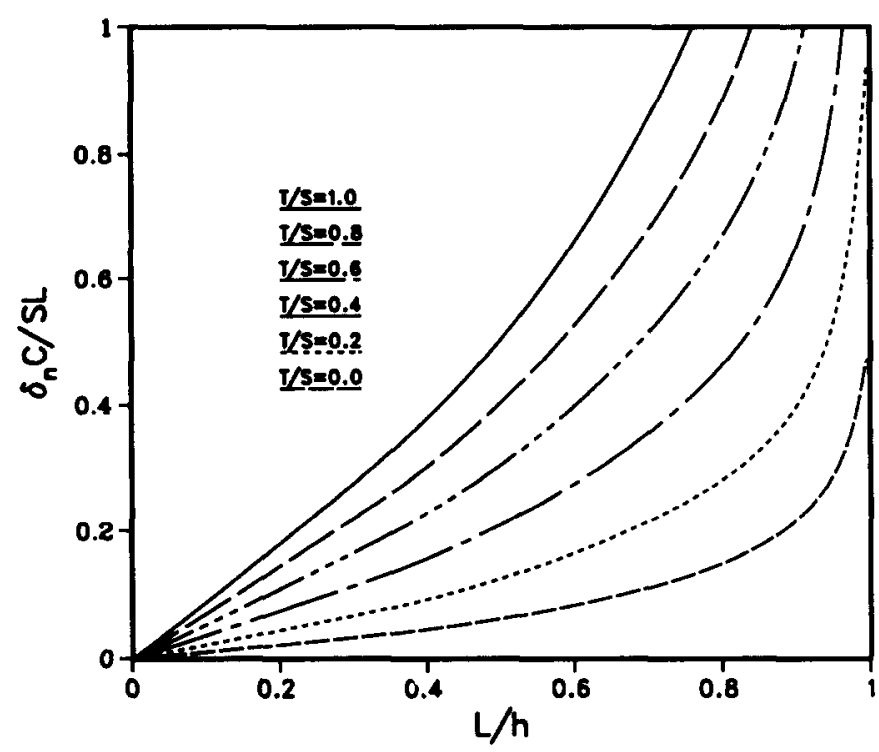

FIG. 2. The relative normal displacement at far field vs ratio of crack length to spacing for various tensile/shear loads $(\beta=0.5)$.

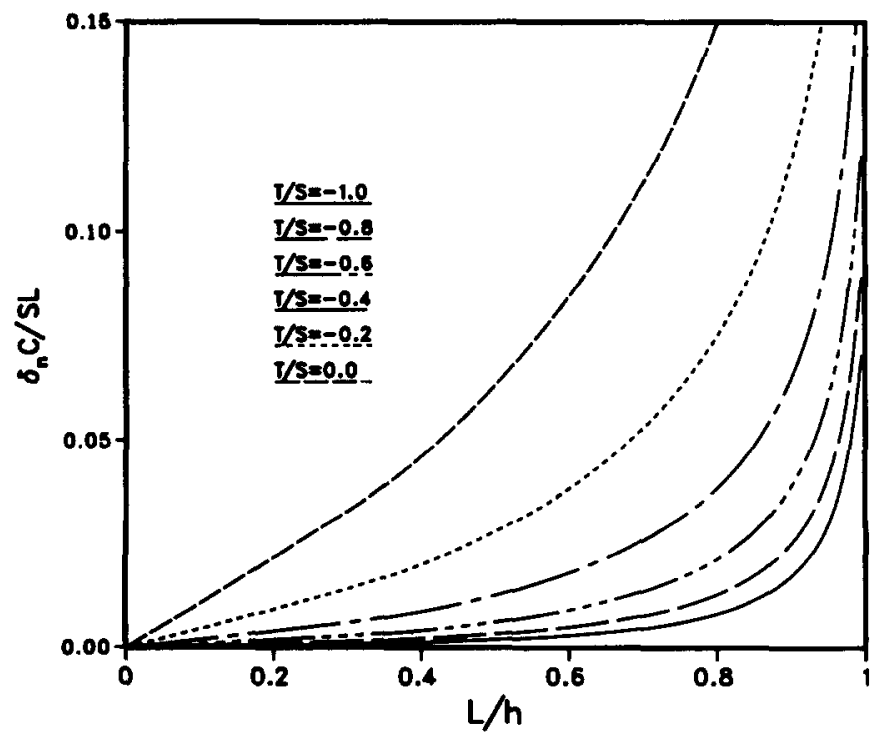

FIG. 3. The relative normal displacement at far field vs ratio of crack length to spacing for various compression/shear loads $(\beta=0.5)$ 
no significant interaction between the cracks when the periodic spacing is greater than about four times the crack length, and the results are as those obtained from a single crack.

Because of the linearity of the average displacement $\delta_{n} C / S L$ with respect to small $L / h$, the quantity $\delta_{n} C h / S L^{2}$ must be constant for $L / h$ less than 0.25 at any given ratio of normal to shear stress $T / S$. This quantity is plotted against $T / S$ for various $L / h$ in Fig. 4. As expected, all curves coincide for $h / L>4$. The curves are different for $h / L<4$ because of crack interaction. We also notice from Fig. 4 the linearity of $\delta_{n} C h / S L^{2}$ for $T / S$ greater than about 0.5 ; the curves become straight lines passing through the origin $(T / S=0)$. This is because the right contact zone reduces rapidly as $T / S$ increases, and it is nearly zero for $T / S>2$ so that its effect on the global fields is negligible. Therefore, $\delta_{n}$ will be proportional to the normal stress $T$ as in the homogeneous case, where there is no coupling between tension and shear. Notice, however, that the normal displacement is not zero for $T / S$ less or equal to zero because of the effect of contact pressure.

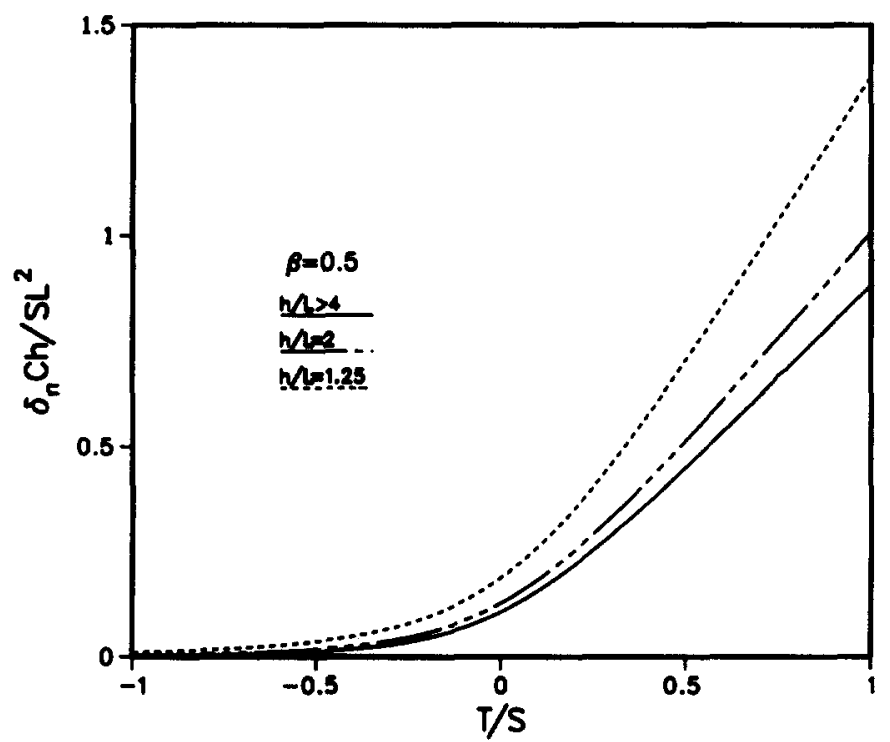

FIG. 4. Normalized relative normal displacement $\delta_{n} C h / S L^{2}$ vs ratio of tension to shear $T / S$ for $h / L>4, h / L=2$ and $h / L=1.25(\beta=0.5)$.

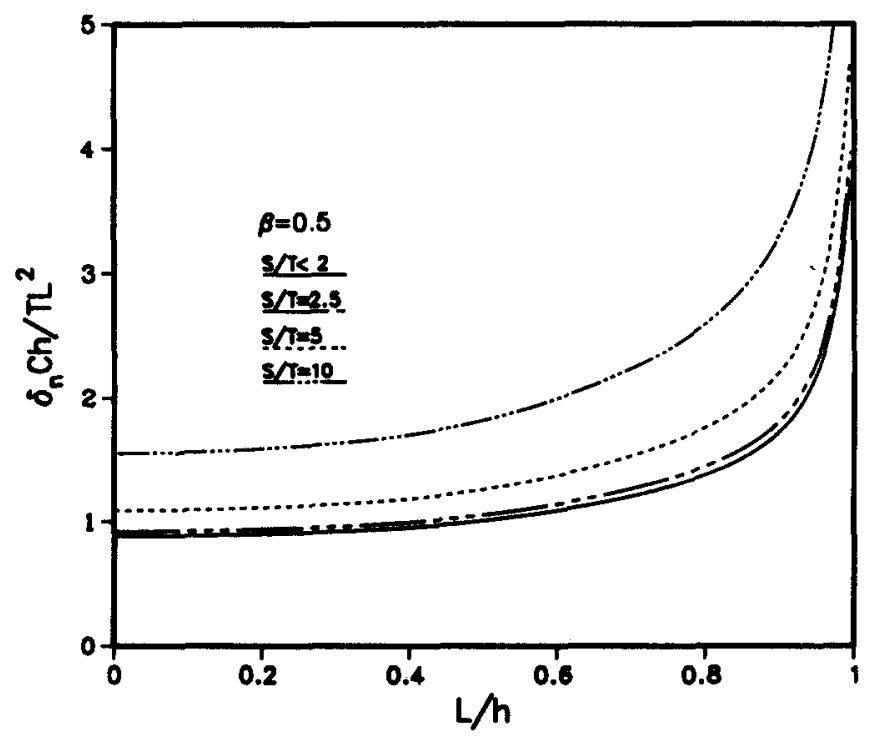

Fig. 5. Normalized relative normal displacement $\delta_{n} C h / T L^{2}$ vs $L / h$ for various ratios of shear to tension $S / T(\beta=0.5)$. 
Figure 4 also implies that $\delta_{n} C h / T L^{2}$ must be constant for large $T / S$ at any given $h / L$. Therefore we plot this quantity against $L / h$ in Fig. 5. For $S / T<2$ all curves coincide as expected. Deviations from this curve are the result of the existence of the contact zone and hence of the coupling between shear and tension.

The effect of the parameter $\beta$ is shown in Fig. 6 for $h / L>4$. For tension/shear combinations the quantity $\delta_{n} \mathrm{Ch} / S L^{2}$ deviates very little from the homogeneous case.

Figure 7 shows the relative tangential displacement $\delta_{t} C / S L$ with respect to $L / h$ for various combinations of normal and shear stresses. Comparing these figures with Figs 2 and 3 , it is seen that the effect of tension on tangential displacement or shear compliance is much smaller than that of shear on normal displacement or normal compliance. Similarly, the shear compliance is linear for small $L / h ; \delta_{t} C h / S L^{2}$ is constant for $L / h$ less than 0.25 at any given $T / S$. For $h / L>4$ all curves coincide in Fig. 8, and for $h / L<4$ the effect of the

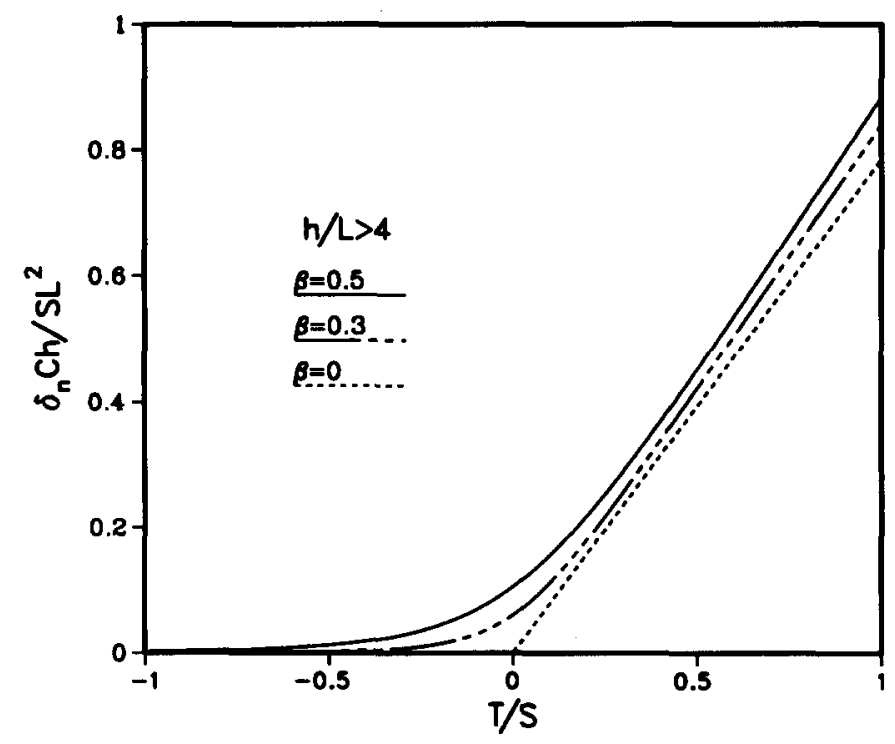

FIG. 6. Normalized relative normal displacement $\delta_{n} C h / S L^{2}$ vs ratio of tension to shear $T / S$ for $h / L>4$ and $\beta=0.5, \beta=0.3, \beta=0$.

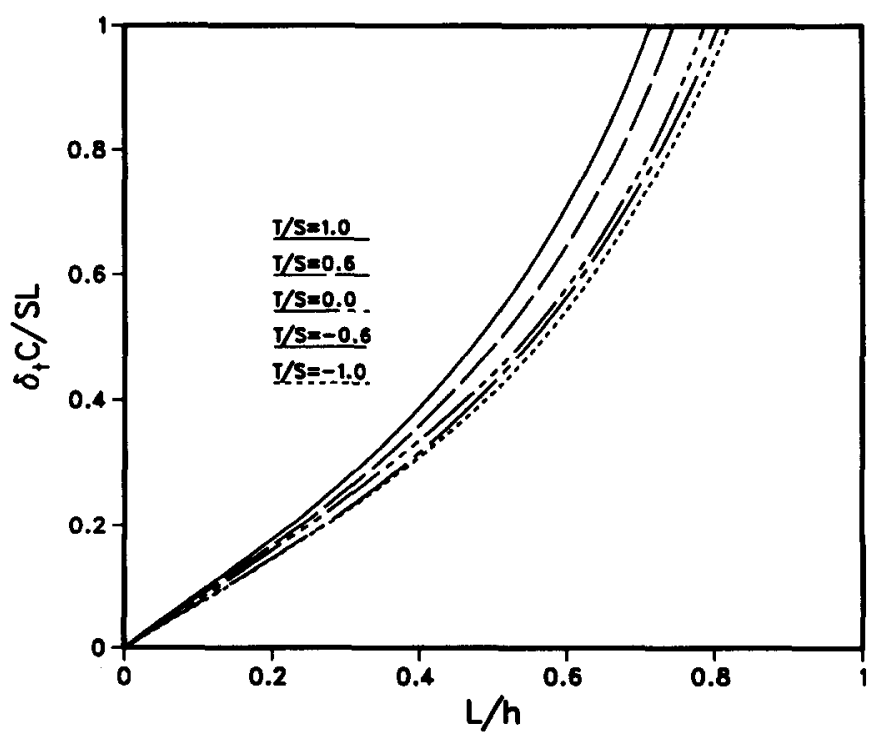

Fig. 7. The relative tangential displacement at far field vs ratio crack length to spacing for various normal/shear loads $(\beta=0.5)$. 
existence of the contact zone results in deviations from this curve. It is also seen from Fig. 8 that for both large tension/shear and compression/shear loads, the curves level out. This is because for large tensile loads the crack is almost fully open, and the small contact zones will not have much effect on displacement. For large compressive loads, the crack is almost fully closed and a further increase in the normal load will not affect the displacement.

The effect of the parameter $\beta$ is shown in Fig. 9 for $h / L>4$. For compression/shear combinations the effect of $\beta$ on the quantity $\delta_{t} C h / S L^{2}$ is negligible.

In general, it can be concluded that interface cracks have significant interaction only when the spacing between the cracks is less than about four times the crack length. The interaction is negligible when the spacing is greater than four times the crack length. The effects of contact zones, and therefore the coupling between tension and shear which

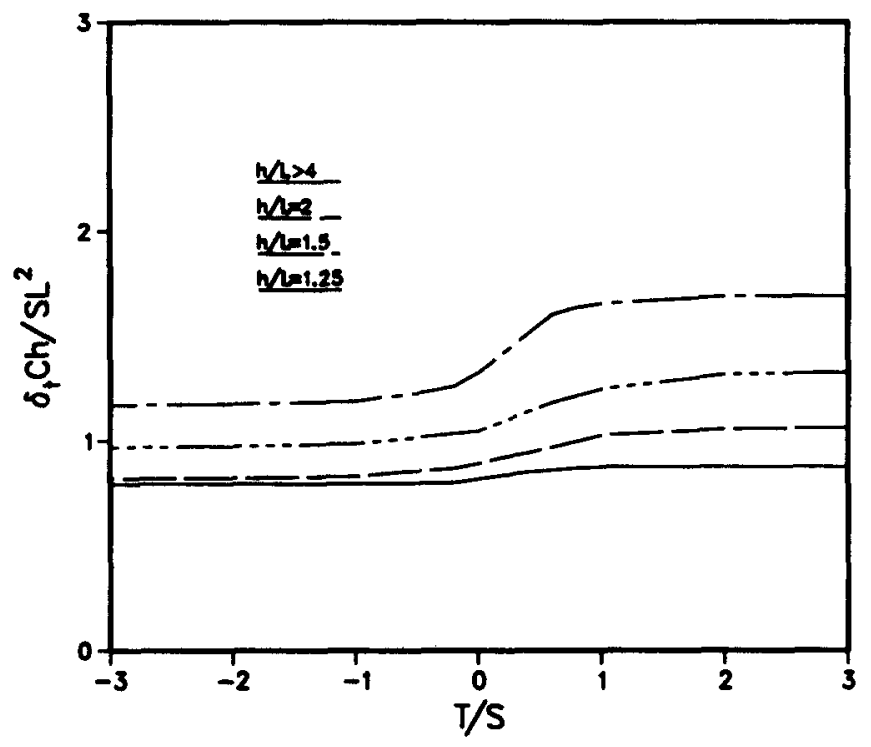

Fig. 8. Normalized relative tangential displacement $\delta_{l} C h / S L^{2}$ vs ratio of tension to shear $T / S$ for $h / L>4, h / L=2$ and $h / L=1.25(\beta=0.5)$.

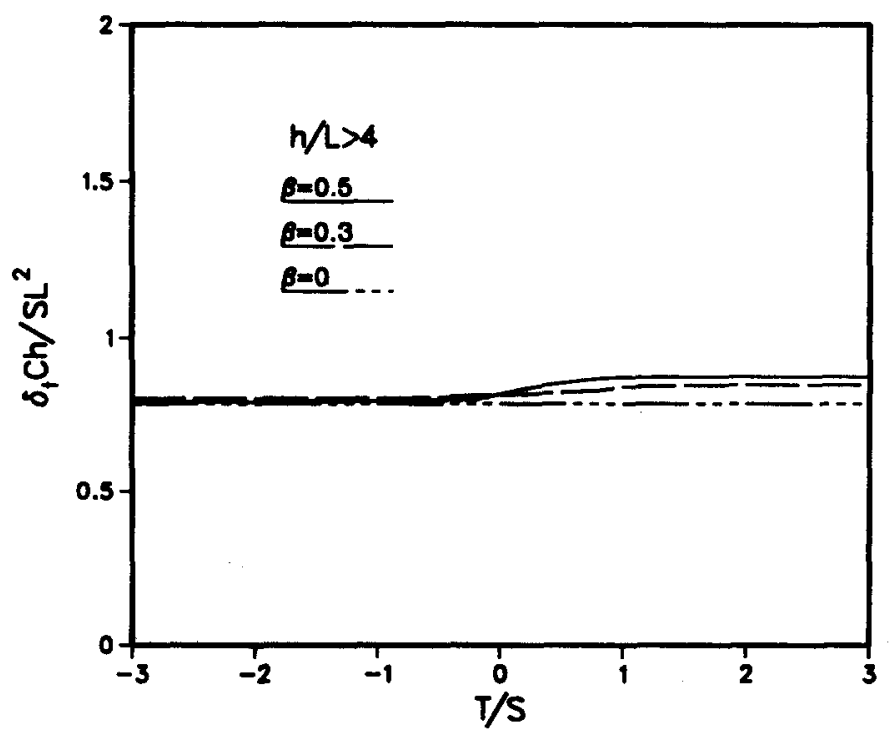

FIG. 9. Normalized relative tangential displacement $\delta_{t} C h / S L^{2}$ vs ratio of tension to shear $T / S$ for $h / L>4$, and $\beta=0.5, \beta=0.3, \beta=0$. 
contributes to the nonlinearity of the problem, are significant only when the normal load is small compared with the shear load.

\section{REFERENCES}

1. A. H. England, A crack between dissimilar media. J. appl. Mech. 32, 400 (1965)

2. M. CoMninou, The interface crack. J. appl. Mech. 44, 631 (1977).

3. R. J. Kerans, R. S. Hay, N. J. Pagano and T. A. Parthasarathy, The role of the fiber-matrix interface in ceramic composites. Ceram. Bull. 68, 429 (1989).

4. M. COMNINOU, The interface crack in a shear field. J. appl. Mech. 45, 287 (1978)

5. M. COMNINOU and D. SCHMUESER, The interface crack in a combined tension-compression and shear field. J. appl. Mech. 46, 345 (1979).

6. D. SCHMUESER and M. COMNINOU, The periodic array of interface cracks and their interaction. Int. J. Struct. 15, 927 (1979).

7. S. P. Timoshenko and J. N. Goodier, Theory of Elasticity, 3rd edn. McGraw-Hill, New York (1970); Il Nuovo cimento, ser. 2, Vols 6-10 (1872).

8. F. ERDOGAN and G. GUPTA, On the numerical solution of singular integral equations. Q. appl. Math. 29, 525 (1972).

9. N. I. MuSKelishviLI, Singular Integral Equations. Noordhoff, Leyden (1953). 